\title{
Spectroscopic orbits and variations of RS Ophiuchi
}

\author{
E. Brandi ${ }^{1,2,3}$, C. Quiroga ${ }^{1,2}$, J. Mikołajewska ${ }^{4}$, O. E. Ferrer ${ }^{1,5}$, and L. G. García ${ }^{1,2}$ \\ 1 Facultad de Ciencias Astronómicas y Geofísicas, Universidad Nacional de La Plata (UNLP), Argentina \\ e-mail: ebrandi@fcaglp.unlp.edu.ar \\ 2 Instituto de Astrofísica de La Plata (CCT La Plata-CONICET-UNLP), Argentina \\ 3 Comisión de Investigaciones Científicas de la Provincia de Buenos Aires (CIC), Argentina \\ ${ }^{4}$ Copernicus Astronomical Center, Warsaw, Poland \\ e-mail:mikolaj@camk.edu.pl \\ 5 Consejo Nacional de Investigaciones Científicas y Técnicas (CONICET), Argentina
}

Received 25 November 2008 / Accepted 25 January 2009

\section{ABSTRACT}

\begin{abstract}
Aims. The aims of our study are to improve the orbital elements of the giant and to derive the spectroscopic orbit for the white dwarf companion of the symbiotic system RS Oph. Spectral variations related to the 2006 outburst are also studied.

Methods. We performed an analysis of about seventy optical and near infrared spectra of RS Oph that were acquired between 1998 and June 2008. The spectroscopic orbits were obtained by measuring the radial velocities of the cool component absorption lines and the broad $\mathrm{H} \alpha$ emission wings, which seem to be associated with the hot component. A set of cF-type absorption lines were also analyzed for a possible connection with the hot component motion.

Results. A new period of 453.6 days and a mass ratio, $q=M_{\mathrm{g}} / M_{\mathrm{h}}=0.59 \pm 0.05$ were determined. Assuming a massive white dwarf as the hot component $\left(M_{\mathrm{h}}=1.2-1.4 M_{\odot}\right)$ the red giant mass is $M_{\mathrm{g}}=0.68-0.80 M_{\odot}$ and the orbit inclination, $i=49^{\circ}-52^{\circ}$. The cF-type lines are not associated with either binary component, and are most likely formed in the material streaming towards the hot component. We also confirm the presence of the Li I doublet in RS Oph and its radial velocities fit very well to the M-giant radial velocity curve. Regardless of the mechanism involved to produce lithium, its origin is most likely from within the cool giant rather than material captured by the giant at the time of the nova explosion.

The quiescent spectra reveal a correlation of the HI and He I emission line fluxes with the monochromatic magnitudes at $4800 \AA$, indicating that the hot component activity is responsible for those flux variations. We also discuss the spectral characteristics around 54-55 and 240 days after the 2006 outburst. In April 2006 most of the emission lines present a broad pedestal with a strong and narrow component at about $-20 \mathrm{~km} \mathrm{~s}^{-1}$ and two other extended emission components at -200 and $+150 \mathrm{~km} \mathrm{~s}^{-1}$. These components could originate in a bipolar gas outflow supporting the model of a bipolar shock-heated shell expanding through the cool component wind perpendicularly to the binary orbital plane. Our observations also indicate that the $\mathrm{cF}$ absorption system was disrupted during the outburst, and restored about 240 days after the outburst, which is consistent with the resumption of accretion.
\end{abstract}

Key words. stars: binaries: symbiotic - stars: novae, cataclysmic variables - stars: individual: RS Oph - techniques: spectroscopic

\section{Introduction}

RS Oph is a recurrent symbiotic nova in which a white dwarf near the Chandrasekhar limit orbits inside the outer wind of a red giant. The system has had numerous recorded outbursts (1898, 1933, 1958, 1967, 1985). The most recent outburst began on 2006 February 12 and multifrequency observations confirmed the current model for the outburst in which the massive white dwarf accretes material from the red giant until a thermonuclear runaway ensues and high velocity gas is ejected from the white dwarf. Because the white dwarf is orbiting inside the outer layers/wind of the red giant, a strong shock structure is established around the system.

For RS Oph, Garcia (1986) made the first orbital period estimate of about 230 days, based on eight radial velocity measurements. Two sets of lines were considered: $M$ star absorption features and absorption cores of Fe II emission lines due to a shell. He could not, however, derive the mass ratio. Oppenheimer \& Mattei (1993) analysed intervals between outbursts in the AAVSO visual light curve and found the strongest periods ranged between 892 and 2283 days. Dobrzycka \& Kenyon (1994, hereafter DK94) continued monitoring the radial velocities of RS Oph during quiescence. They also separated their echelle spectra into sets of M-type and A-type absorption lines, both of which resulted in a period of 460 days. The Atype radial velocity curve was shifted by 0.37 relative to the $\mathrm{M}$ giant solution and it was not associated with the hot component motion. They concluded that their results are more reasonable if they only considered the circular orbit of the cool giant. DK94 searched for periodic variations in photometric data of RS Oph collected during 1971-1983 and after the 1985 outburst. For both time intervals they found the light curve to be the superposition of a longer period variation $(P=2178$ days $)$ and a shorter variation ( $P=508$ days $)$. The longer period was in agreement with $P=2283$ days given by Oppenheimer \& Mattei (1993) but it did not appear in their radial velocity analysis so it could not be associated with the orbital motion. Fifteen infrared radial velocities were used by Fekel et al. (2000, hereafter FJHS00) combined with the 47 optical velocities of DK94 in order to improve the orbital elements of the giant. FJHSO0 fitted a new period of 455.72 days for a circular solution and eccentric orbits were rejected applying the test of Lucy \& Sweeney (1971).

In this study, we have collected spectroscopic data of RS Oph from September 1998 until October 2006 and two Feros spectra 
taken on May and June 2008 were also included in our analysis. Our observation closest to the eruption was obtained in April 2006.

We describe our observations in Sect. 2; in Sect. 3 we determine double-line spectroscopic orbits for RS Oph; in Sect. 4 spectral characteristics observed during quiescence are presented; in Sect. 5 we describe spectral changes during the active phase at 54-55 and 240 days after eruption and finally a brief discussion of our results and conclusions are given in Sect. 6.

\section{Observations}

\subsection{Spectroscopy}

Spectroscopic observations of RS Oph were performed with the $2.15 \mathrm{~m}$ "Jorge Sahade" telescope of CASLEO' (San Juan, Argentina), during 1998-2006. 65 spectra with resolution 12000-15000 were obtained with a REOSC echelle spectrograph using a Tek CCD $1024 \times 1024$ pixels. The CCD data were reduced with IRAF $^{2}$ packages CCDRED and ECHELLE and all the spectra were measured using the SPLOT task within IRAF.

To obtain the flux calibration, standard stars from Hamuy et al. (1992) and Hamuy et al. (1994) were observed each night. A comparison of the spectra of the standards suggests that the flux calibration errors are about $20 \%$ in the central part of each echelle order.

Two high resolution spectra were acquired in May and June 2008 with the Feros spectrograph mounted at the $2.2 \mathrm{~m}$ telescope at ESO, La Silla (Chile). An EEV CCD-44 with $2048 \times 4096$ pixels was used as the detector with a pixel size of $15 \times 15 \mu \mathrm{m}$ and resolving power of $R=60000$.

\section{Spectroscopic orbits}

Spectroscopic orbits of RS Oph were calculated using high resolution spectra collected at CASLEO during the period 19982006.

To obtain the radial velocities of the red giant we have measured M-type absorptions in the region $\lambda \sim 6000-8000 \AA$, corresponding to Fe I, Ti I, Ni I, Si I, O I, Zr I, Co I, V I, Mg I and Gd II. We have also identified and measured absorption lines of singly ionized elements in the blue region of our spectra ( $\lambda \sim 4000-5800 \AA$ ), which resemble spectra of A-F supergiants. This set of absorption lines, called cF-absorptions, are believed to be linked to the hot companion (e.g. Mikołajewska \& Kenyon 1992; Quiroga et al. 2002; Brandi et al. 2005). These are basically the same lines that are called A-type absorption lines by DK94. As the Fe II lines show very variable and complex profiles with emission components, only the stronger Ti II absorption lines were considered in our orbital solutions.

In both cases individual radial velocities were obtained by a Gaussian fit of the line profiles. A mean value was calculated for each spectrum and the resulting heliocentric velocities together with their standard errors and with the number of measured lines are given in Table 1 . The published radial velocities of the M-giant were also included in the table.

\footnotetext{
1 Complejo Astronómico El Leoncito operated under agreement between the Consejo Nacional de Investigaciones Científicas y Técnicas de la República Argentina and the National Universities of La Plata, Córdoba and San Juan.

2 IRAF is distributed by the National Optical Astronomy Observatories, which is operated by the association of Universities for Research in Astronomy, INC under contract to the National Science Foundation.
}

Table 2 lists the resulting orbital solutions for the M-giant using only our radial velocities as well as our radial velocities combined with the DK94 and FJHSO0 data and considering in this case weighted values of radial velocities. We have applied a weight of 1 to the FJHS00 data and weights $0.33,0.67$ and 1 when the errors are larger than 4, between 2 and 3 and less than 2, respectively, for both the DK94 and our data. But we noted that the weighted solutions show no important differences when a weight of 1 is applied to all the data.

A new orbital period of $453.6 \pm 0.4$ days was determined. An elliptical orbit $(e=0.14 \pm 0.03)$ fits the measured velocities slightly better than a circular one (see Fig. 1a).

We have also determined the radial velocities from the broad emission wings of $\mathrm{H} \alpha$ which should reflect the motion of the hot component if the wings were formed in the inner region of the accretion disk or in an extended envelope near the hot component. For this we have used the method outlined by Schneider \& Young (1980, for more details see Quiroga et al. 2002). This method consists of convolving the data with two identical Gaussian bandpasses whose centers have a separation of $2 b$ with standard deviation $\sigma$. We have experimented with a range of values for $b$ and $\sigma$ fixed at $3 \AA$, for which we have obtained the circular orbit solutions. Figure 2 shows the dependence of the standard error $\sigma_{K}$ of the orbital semi-amplitude $K$, and the $\sigma_{K} / K$ ratio, as a function of $b$. It is noticeable that $\sigma_{K}$ and $\sigma_{K} / K$ increases sharply for $b$ larger than $\sim 7 \AA$. We can attribute these large standard errors to the velocity measurements dominated by the noise in the continuum rather than by the extreme high velocity wings of the line profile. We have therefore adopted $b=7 \AA$ as the best value. The heliocentric radial velocities of the broad emission wings of $\mathrm{H} \alpha$ are included in Table 1.

The broad emission line wings of $\mathrm{H} \alpha$ show a mean velocity similar to the red giant systemic velocity (see Fig. 1b). They are clearly in antiphase with the M-giant curve, which suggests that they are really formed in a region very near to the hot component.

Finally we have calculated the combined orbital solutions from the M-giant absorptions and from the $\mathrm{H} \alpha$ broad emission wings (see Table 2). From a comparison of the variances of the velocities of the two individual solutions, weights of 0.64 and 1 were given to the $\mathrm{H} \alpha$ wings and the M-giant velocities, respectively. Leaving the period as a free parameter and taking a circular orbit, the best solution corresponds to a similar period of $454.1 \pm 0.41$ days and adopting the period 453.6 days, the best combined solution leads to a lower eccentricity, $e=0.04 \pm 0.03$. Moreover, we have considered binned data of radial velocities taking phase intervals of 0.05 , and as it is shown in Table 2, very similar results were obtained. These solutions show clear antiphase variations of both sets of radial velocities and the systemic velocity agrees very well with that of the cool component orbital solutions. The combined unbinned and binned solutions are shown in Figs. 1c and d, respectively.

Using the combined circular solutions for unbinned data, we have estimated the mass ratio $q=M_{\mathrm{g}} / M_{\mathrm{h}}=0.59 \pm 0.05$, the component masses $M_{\mathrm{g}} \sin ^{3} i=0.35 M_{\odot}$ and $M_{\mathrm{h}} \sin ^{3} i=0.59 M_{\odot}$, and the binary separation $a \sin i=240 R_{\odot}$. Assuming that the hot component is a massive white dwarf close to the Chandrasekhar limit $\left(M_{\mathrm{h}}=1.2-1.4 M_{\odot}\right)$, we estimate the red giant mass, $M_{\mathrm{g}}=$ $0.68-0.80 M_{\odot}$ and the orbit inclination, $i=49^{\circ}-52^{\circ}$, which is consistent with the absence of eclipses in both, the optical light curve and the $\mathrm{HI}$ and $\mathrm{He}$ I emission line fluxes in our spectra.

A lower limit for the mass ratio can be also derived from the ratio of the rotational velocity to the orbital semiamplitude 
Table 1. Radial velocities of the M-giant component, $\mathrm{H} \alpha$-wings and $\mathrm{cF}$-absorptions in RS Oph.

\begin{tabular}{|c|c|c|c|c|c|c|c|}
\hline \multirow{2}{*}{$\begin{array}{l}\text { JD } \\
2400000+\end{array}$} & \multirow{2}{*}{ Phase $(\dagger)$} & \multicolumn{2}{|c|}{ M-giant } & \multicolumn{2}{|c|}{$\mathrm{H} \alpha$-wings } & \multirow{2}{*}{$\begin{array}{c}\text { cF-abs } \\
\mathrm{RV}\left(\mathrm{km} \mathrm{s}^{-1}\right)\end{array}$} & \multirow{2}{*}{ Source } \\
\hline & & $\mathrm{RV}\left(\mathrm{km} \mathrm{s}^{-1}\right)$ & $(\mathrm{O}-\mathrm{C})$ & $\mathrm{RV}\left(\mathrm{km} \mathrm{s}^{-1}\right)$ & $(\mathrm{O}-\mathrm{C})$ & & \\
\hline 45035.038 & -0.019 & -41.0 & 0.0 & & & & DK94 \\
\hline 45063.017 & 0.043 & -32.0 & 2.5 & & & & DK94 \\
\hline 45063.963 & 0.045 & -35.0 & -0.7 & & & & DK94 \\
\hline 45097.923 & 0.120 & -27.4 & -0.1 & & & & DK94 \\
\hline 45426.977 & 0.845 & -41.1 & 11.9 & & & & DK94 \\
\hline 45426.993 & 0.845 & -41.6 & 11.4 & & & & DK94 \\
\hline 45513.743 & 0.037 & -36.6 & -1.5 & & & & DK94 \\
\hline 46538.993 & 0.297 & -29.0 & -6.8 & & & & DK94 \\
\hline 46540.952 & 0.301 & -32.0 & -9.6 & & & & DK94 \\
\hline 46602.834 & 0.438 & -40.7 & -8.9 & & & & DK94 \\
\hline 46605.743 & 0.444 & -39.3 & -6.8 & & & & DK94 \\
\hline 46608.764 & 0.451 & -39.4 & -6.3 & & & & DK94 \\
\hline 46635.720 & 0.510 & -37.6 & 1.8 & & & & DK94 \\
\hline 46691.608 & 0.633 & -54.1 & -3.0 & & & & DK94 \\
\hline 46867.010 & 0.020 & -38.0 & -1.1 & & & & DK94 \\
\hline 46891.957 & 0.075 & -34.9 & -3.7 & & & & DK94 \\
\hline 46895.935 & 0.084 & -33.9 & -3.5 & & & & DK94 \\
\hline 46918.913 & 0.134 & -32.2 & -6.0 & & & & DK94 \\
\hline 46929.994 & 0.159 & -27.7 & -3.2 & & & & DK94 \\
\hline 46953.833 & 0.211 & -23.5 & -1.3 & & & & DK94 \\
\hline 46979.733 & 0.268 & -25.8 & -4.1 & & & & DK94 \\
\hline 47312.835 & 0.003 & -39.3 & -0.6 & & & & DK94 \\
\hline 47313.822 & 0.005 & -38.6 & -0.1 & & & & DK94 \\
\hline 47486.561 & 0.386 & -38.2 & -11.0 & & & & DK94 \\
\hline 47719.764 & 0.900 & -59.2 & -10.2 & & & & DK94 \\
\hline 47777.656 & 0.028 & -36.0 & 0.1 & & & & DK94 \\
\hline 47787.683 & 0.050 & -37.4 & -3.6 & & & & DK94 \\
\hline 47811.576 & 0.102 & -30.5 & -1.8 & & & & DK94 \\
\hline 47967.019 & 0.445 & -29.5 & 3.1 & & & & DK94 \\
\hline 47992.928 & 0.502 & -43.9 & -5.4 & & & & DK94 \\
\hline 48016.933 & 0.555 & -44.5 & -0.4 & & & & DK94 \\
\hline 48048.826 & 0.625 & -54.3 & -3.8 & & & & DK94 \\
\hline 48349.954 & 0.289 & -22.8 & -0.8 & & & & DK94 \\
\hline 48368.857 & 0.331 & -21.2 & 2.4 & & & & DK94 \\
\hline 48400.832 & 0.401 & -20.1 & 8.4 & & & & DK94 \\
\hline 48411.892 & 0.426 & -30.3 & 0.4 & & & & DK94 \\
\hline 48429.805 & 0.465 & -38.5 & -3.9 & & & & DK94 \\
\hline 48695.025 & 0.050 & -39.5 & -5.8 & & & & DK94 \\
\hline 48723.000 & 0.112 & -30.6 & -2.7 & & & & DK94 \\
\hline 48754.922 & 0.182 & -28.9 & -5.6 & & & & DK94 \\
\hline 48782.831 & 0.244 & -34.3 & -12.7 & & & & DK94 \\
\hline 48816.771 & 0.318 & -29.5 & -6.5 & & & & DK94 \\
\hline 48872.718 & 0.442 & -34.7 & -2.5 & & & & DK94 \\
\hline 48909.584 & 0.523 & -44.4 & -3.6 & & & & DK94 \\
\hline 49106.948 & 0.958 & -42.8 & 0.7 & & & & DK94 \\
\hline 49136.907 & 0.024 & -36.6 & -0.2 & & & & DK94 \\
\hline 49174.784 & 0.108 & -30.4 & -2.1 & & & & DK94 \\
\hline 49998.639 & 0.924 & -49.1 & -2.3 & & & & FJHS00 \\
\hline 50162.905 & 0.286 & -18.0 & 4.0 & & & & FJHS00 \\
\hline 50254.723 & 0.488 & -33.5 & 3.6 & & & & FJHS00 \\
\hline 50320.643 & 0.634 & -48.5 & 2.6 & & & & FJHSO0 \\
\hline 50387.593 & 0.781 & -61.9 & -6.4 & & & & FJHS00 \\
\hline 50568.854 & 0.181 & -27.5 & -4.2 & & & & FJHS00 \\
\hline 50627.799 & 0.311 & -19.3 & 3.4 & & & & FJHS00 \\
\hline 50751.574 & 0.584 & -49.6 & -2.7 & & & & FJHS00 \\
\hline 50933.851 & 0.986 & -42.1 & -1.5 & & & & FJHSOO \\
\hline 50981.817 & 0.091 & -27.5 & 2.2 & & & & FJHSO0 \\
\hline 50983.747 & 0.096 & -26.8 & 2.5 & & & & FJHS00 \\
\hline 51051.677 & 0.245 & -19.7 & 1.9 & & & & FJHSO0 \\
\hline 51064.570 & 0.274 & & & -40.7 & 8.0 & & CASLEO \\
\hline 51066.532 & 0.278 & $-18.4 \pm 8.3(4)$ & 3.4 & & & $-23.3 \pm 1.2(3)$ & CASLEO \\
\hline 51069.467 & 0.285 & $-15.3 \pm 5.4(4)$ & 6.6 & & & $-28.7 \pm 3.5(6)$ & CASLEO \\
\hline 51069.537 & 0.285 & $-13.9 \pm 7.5(4)$ & 8.0 & & & $-24.4 \pm 4.9(5)$ & CASLEO \\
\hline 51069.571 & 0.285 & $-18.6 \pm 5.7(5)$ & 3.3 & & & $-26.7 \pm 3.6(5)$ & CASLEO \\
\hline 51106.592 & 0.367 & -27.4 & -1.7 & & & & FJHS00 \\
\hline
\end{tabular}


Table 1. continued.

\begin{tabular}{|c|c|c|c|c|c|c|c|}
\hline JD & Phase $(\dagger)$ & M-giant & & $\mathrm{H} \alpha-\mathrm{w}$ & & $\mathrm{cF}-\mathrm{abs}$ & Source \\
\hline $2400000+$ & & $\mathrm{RV}\left(\mathrm{km} \mathrm{s}^{-1}\right)$ & $(\mathrm{O}-\mathrm{C})$ & $\mathrm{RV}\left(\mathrm{km} \mathrm{s}^{-1}\right)$ & $(\mathrm{O}-\mathrm{C})$ & $\mathrm{RV}\left(\mathrm{km} \mathrm{s}^{-1}\right)$ & \\
\hline 51294.889 & 0.782 & -63.5 & -8.0 & & & & FJHS00 \\
\hline 51324.666 & 0.847 & $-55.3 \pm 5.7(24)$ & -2.5 & -32.8 & -2.5 & $-30.2 \pm 3.7$ & CASLEO \\
\hline 51324.678 & 0.847 & $-53.1 \pm 2.1(21)$ & -0.3 & -34.2 & -3.9 & $-30.1 \pm 2.5(5)$ & CASLEO \\
\hline 51324.690 & 0.847 & $-54.6 \pm 2.0(20)$ & -1.8 & -32.1 & 1.8 & $-24.9 \pm 3.3(3)$ & CASLEO \\
\hline 51363.664 & 0.933 & -45.0 & 0.9 & & & & FJHSO0 \\
\hline 51422.584 & 0.063 & $-33.6 \pm 1.4(9)$ & -1.2 & -49.6 & -7.2 & $-34.4 \pm 1.2(7)$ & CASLEO \\
\hline 51422.599 & 0.063 & $-29.5 \pm 2.1(6)$ & 2.9 & -46.2 & -3.8 & $-36.2 \pm 2.5(7)$ & CASLEO \\
\hline 51628.861 & 0.518 & $-37.5 \pm 1.7(31)$ & 2.7 & -39.7 & -2.0 & $-42.4 \pm 1.8(7)$ & CASLEO \\
\hline 51628.870 & 0.518 & $-38.4 \pm 1.7(28)$ & 1.8 & -38.3 & -0.6 & $-44.0 \pm 2.0(6)$ & CASLEO \\
\hline 51629.850 & 0.520 & $-39.6 \pm 2.2(22)$ & 0.9 & -38.9 & -1.3 & $-40.6 \pm 3.1(7)$ & CASLEO \\
\hline 51629.859 & 0.520 & $-39.8 \pm 1.5(28)$ & 0.7 & -38.9 & -1.3 & $-43.6 \pm 2.4(7)$ & CASLEO \\
\hline 51629.868 & 0.520 & $-39.6 \pm 1.9(26)$ & 0.9 & -38.9 & -1.3 & $-43.9 \pm 2.7(6)$ & CASLEO \\
\hline 51629.876 & 0.520 & $-40.7 \pm 2.0(26)$ & -0.2 & -36.0 & 1.6 & $-43.5 \pm 3.3(6)$ & CASLEO \\
\hline 51630.809 & 0.522 & $-37.6 \pm 1.8(27)$ & 3.1 & -33.1 & 4.4 & $-47.2 \pm 0.5(4)$ & CASLEO \\
\hline 51630.832 & 0.522 & $-39.0 \pm 2.2(22)$ & 1.7 & -31.5 & 6.0 & $-46.7 \pm 3.4(5)$ & CASLEO \\
\hline 51754.660 & 0.795 & $-59.4 \pm 3.8(6)$ & -4.3 & -27.4 & 1.5 & $-29.4 \pm 1.6(7)$ & CASLEO \\
\hline 51754.669 & 0.795 & $-58.7 \pm 3.8(6)$ & -3.6 & -29.1 & -0.2 & $-30.1 \pm 2 .(6)$ & CASLEO \\
\hline 51754.680 & 0.795 & $-57.7 \pm 4.1(6)$ & -2.6 & -27.8 & 1.1 & $-26.5 \pm 2.5(7)$ & CASLEO \\
\hline 51790.615 & 0.874 & $-52.4 \pm 2.9(10)$ & -1.4 & -34.2 & -2.9 & $-18.6 \pm 0.7(7)$ & CASLEO \\
\hline 51790.623 & 0.874 & $-53.1 \pm 2.7(9)$ & -2.1 & -34.0 & -2.7 & $-19.1 \pm 1.1(6)$ & CASLEO \\
\hline 51790.630 & 0.874 & $-50.2 \pm 3.8(9)$ & 0.8 & -33.9 & -2.6 & $-20.2 \pm 1.7(7)$ & CASLEO \\
\hline 51791.616 & 0.877 & $-48.4 \pm 1.1(42)$ & 2.4 & -23.2 & 8.2 & & CASLEO \\
\hline 51791.623 & 0.877 & $-46.7 \pm 1.3(39)$ & 4.2 & -23.7 & 7.7 & & CASLEO \\
\hline 51791.630 & 0.877 & $-47.4 \pm 1.3(39)$ & 3.5 & -23.5 & 7.9 & & CASLEO \\
\hline 51798.524 & 0.892 & $-54.8 \pm 2.1(7)$ & -5.1 & -29.0 & 3.2 & $-18.6 \pm 4.0(7)$ & CASLEO \\
\hline 51798.533 & 0.892 & $-54.8 \pm 2.5(6)$ & -5.1 & -26.9 & 5.3 & $-22.1 \pm 1.4(6)$ & CASLEO \\
\hline 51824.502 & 0.949 & $-40.8 \pm 1.3(10)$ & 3.6 & -29.1 & 6.2 & $-32.7 \pm 1.1(7)$ & CASLEO \\
\hline 51824.511 & 0.949 & $-38.5 \pm 1.9(9)$ & 5.9 & -28.9 & 6.4 & $-32.7 \pm 1.6(7)$ & CASLEO \\
\hline 51824.520 & 0.949 & $-41.4 \pm 1.9(10)$ & 3.0 & -28.5 & 6.8 & $-33.7 \pm 1.3(6)$ & CASLEO \\
\hline 51827.511 & 0.956 & $-40.4 \pm 1.2(39)$ & 3.3 & -30.0 & 5.7 & & CASLEO \\
\hline 51827.518 & 0.956 & $-39.0 \pm 1.1(38)$ & 4.7 & -30.2 & 5.5 & & CASLEO \\
\hline 51827.526 & 0.956 & $-41.2 \pm 1.3(38)$ & 2.5 & -30.2 & 5.5 & & CASLEO \\
\hline 52016.843 & 0.373 & $-19.8 \pm 2.4(11)$ & 6.4 & -51.5 & -5.4 & $-32.4 \pm 1.5(7)$ & CASLEO \\
\hline 52016.853 & 0.373 & $-24.8 \pm 1.8(6)$ & 1.4 & -52.2 & -6.1 & $-32.3 \pm 2.6(7)$ & CASLEO \\
\hline 52016.861 & 0.373 & $-24.3 \pm 4.6(5)$ & 1.9 & -49.4 & -3.3 & $-35.6 \pm 1.6(6)$ & CASLEO \\
\hline 52058.859 & 0.466 & $-36.6 \pm 4.3(9)$ & -1.9 & -34.8 & 6.2 & & CASLEO \\
\hline 52058.867 & 0.466 & $-37.9 \pm 9.7(6)$ & -3.2 & -34.3 & 6.7 & & CASLEO \\
\hline 52058.875 & 0.466 & $-34.0 \pm 2.1(2)$ & 0.7 & -38.3 & 2.7 & & CASLEO \\
\hline 52059.853 & 0.468 & $-33.5 \pm 1.2(36)$ & 1.4 & & & & CASLEO \\
\hline 52059.861 & 0.468 & $-34.0 \pm 1.1(33)$ & 0.9 & -39.0 & 1.9 & & CASLEO \\
\hline 52059.869 & 0.468 & $-34.3 \pm 1.1(32)$ & 0.6 & -40.1 & 0.8 & & CASLEO \\
\hline 52132.575 & 0.628 & $-60.7 \pm 1.2(7)$ & -10.0 & -34.1 & -2.6 & $-41.8 \pm 5.1(6)$ & CASLEO \\
\hline 52132.584 & 0.628 & $-61.0 \pm 2.0(7)$ & -10.3 & -35.0 & -3.5 & $-42.1 \pm 5.1(6)$ & CASLEO \\
\hline 52388.895 & 0.193 & $-26.8 \pm 1.0(89)$ & -4.0 & -50.5 & -2.4 & $-33.5 \pm 0.2(2)$ & CASLEO \\
\hline 52388.914 & 0.193 & $-24.4 \pm 0.95(93)$ & -1.6 & -50.2 & -2.1 & $-26.7 \pm 1.2(2)$ & CASLEO \\
\hline 52529.553 & 0.504 & & & -28.7 & 10.0 & & CASLEO \\
\hline 52531.543 & 0.508 & $-38.7 \pm 1.3(122)$ & 0.5 & -32.7 & 5.7 & & CASLEO \\
\hline 52531.556 & 0.508 & $-37.7 \pm 1.3(123)$ & 1.5 & -33.7 & 4.7 & & CASLEO \\
\hline 52770.858 & 0.036 & $-34.5 \pm 1.6(70)$ & 0.7 & -28.7 & 12.0 & $-26.9 \pm 2.2(3)$ & CASLEO \\
\hline 52770.873 & 0.036 & $-37.2 \pm 1.8(68)$ & -2.0 & -29.2 & 11.5 & $-26.1 \pm 0.4(3)$ & CASLEO \\
\hline 52771.837 & 0.038 & $-36.6 \pm 3.2(29)$ & -1.6 & -35.5 & 5.3 & $-26.9 \pm 1.8(6)$ & CASLEO \\
\hline 52771.846 & 0.038 & $-37.2 \pm 3.3(24)$ & -2.2 & -36.6 & 4.2 & $-25.3 \pm 1.8(6)$ & CASLEO \\
\hline 52772.848 & 0.040 & & & -31.4 & 9.6 & & CASLEO \\
\hline 52772.860 & 0.040 & & & -30.7 & 10.3 & & CASLEO \\
\hline 52887.575 & 0.292 & & & -38.7 & 9.8 & & CASLEO \\
\hline 52890.529 & 0.299 & $-21.6 \pm 0.9(110)$ & 0.7 & -34.4 & 14.0 & & CASLEO \\
\hline 52890.542 & 0.299 & $-23.5 \pm 0.9(121)$ & -1.2 & -34.9 & 13.5 & & CASLEO \\
\hline 53126.836 & 0.820 & $-54.6 \pm 1.4(65)$ & -0.4 & & & $-26.5 \pm 3.4(5)$ & CASLEO \\
\hline 53126.848 & 0.820 & $-54.5 \pm 1.9(55)$ & -0.3 & & & $-24.5 \pm 1.6(6)$ & CASLEO \\
\hline 53127.850 & 0.822 & $-56.1 \pm 1.1(121)$ & -2.0 & -30.9 & -1.4 & & CASLEO \\
\hline 53127.864 & 0.822 & $-54.2 \pm 1.4(119)$ & -0.1 & -32.0 & -2.5 & & CASLEO \\
\hline 53476.884 & 0.592 & $-51.6 \pm 1.7(69)$ & -3.9 & -28.0 & 5.3 & $-43.5 \pm 2.7(3)$ & CASLEO \\
\hline 53476.897 & 0.592 & $-50.4 \pm 1.5(74)$ & -2.7 & -25.4 & 7.9 & $-42.4 \pm 2.4(3)$ & CASLEO \\
\hline 53479.879 & 0.599 & $-55.4 \pm 1.3(91)$ & -7.1 & -36.4 & -3.4 & & CASLEO \\
\hline 53628.562 & 0.926 & $-51.1 \pm 1.8(32)$ & -4.5 & & & $-38.7 \pm 2.7(4)$ & CASLEO \\
\hline
\end{tabular}


Table 1. continued.

\begin{tabular}{|c|c|c|c|c|c|c|c|}
\hline JD & Phase $(\dagger)$ & M-gian & & $\mathrm{H} \alpha$-wi & & cF-abs & Source \\
\hline $2400000+$ & & $\mathrm{RV}\left(\mathrm{km} \mathrm{s}^{-1}\right)$ & $(\mathrm{O}-\mathrm{C})$ & $\mathrm{RV}\left(\mathrm{km} \mathrm{s}^{-1}\right)$ & $(\mathrm{O}-\mathrm{C})$ & $\mathrm{RV}\left(\mathrm{km} \mathrm{s}^{-1}\right)$ & \\
\hline 54021.484 & 0.793 & $-57.2 \pm 2.4(58)$ & -2.0 & -18.7 & 10.2 & $-25.3 \pm 0.4(3)$ & CASLEO \\
\hline 54021.496 & 0.793 & $-57.1 \pm 2.5(66)$ & -1.9 & -20.0 & 8.9 & $-24.2 \pm 2.2(4)$ & CASLEO \\
\hline
\end{tabular}

( $\dagger$ ) We adopt the spectroscopic ephemeris: $T_{\text {conj }}=2445043.54+453.6 \mathrm{E}$.

Table 2. Orbital solutions of RS Oph.

\begin{tabular}{|c|c|c|c|c|c|c|c|c|c|}
\hline Component & $\begin{array}{c}P \\
\text { [days] }\end{array}$ & $\begin{array}{c}K \\
{\left[\mathrm{~km} \mathrm{~s}^{-1}\right]}\end{array}$ & $\begin{array}{c}\gamma_{0} \\
{\left[\mathrm{~km} \mathrm{~s}^{-1}\right]}\end{array}$ & $e$ & $\begin{array}{c}\omega \\
{[\mathrm{deg}]}\end{array}$ & $\begin{array}{c}T_{0}^{(1)} \\
{[\mathrm{JD} 24 \ldots]}\end{array}$ & $\Delta \phi^{(2)}$ & $\begin{array}{l}a \sin i \\
{[\mathrm{AU}]}\end{array}$ & $\begin{array}{l}f(M) \\
{\left[M_{\odot}\right]}\end{array}$ \\
\hline \multicolumn{10}{|l|}{$\overline{M-g i a n t}$} \\
\hline Our data & $452.8 \pm 1.5$ & $19.5 \pm 0.7$ & $-38.0 \pm 0.4$ & $0^{(3)}$ & & 51055.50 & \multirow{4}{*}{0.00} & 0.81 & 0.35 \\
\hline Our data & $453.2 \pm 1.2$ & $20.0 \pm 0.7$ & $-38.7 \pm 0.4$ & $0.10 \pm 0.03$ & $120 \pm 16$ & 51205.95 & & 0.83 & 0.37 \\
\hline $\mathrm{DK}+\mathrm{F}+$ ours & $453.6 \pm 0.4$ & $17.5 \pm 0.6$ & $-39.5 \pm 0.4$ & $0^{(3)}$ & & 45156.94 & & 0.73 & 0.25 \\
\hline $\mathrm{DK}+\mathrm{F}+$ ours & $453.6^{(3)}$ & $18.6 \pm 0.6$ & $-40.2 \pm 0.4$ & $0.14 \pm 0.03$ & $135 \pm 11$ & 44873.23 & & 0.77 & 0.29 \\
\hline \multicolumn{10}{|l|}{ cF-abs } \\
\hline \multirow[t]{2}{*}{ Our data ${ }^{(5)}$} & $453.6^{(3)}$ & $8.0 \pm 1.2$ & $-32.5 \pm 0.9$ & $0^{(3)}$ & & 50493.51 & \multirow[t]{2}{*}{-0.24} & 0.33 & 0.024 \\
\hline & $453.6^{(3)}$ & $10.2 \pm 1.8$ & $-31.8 \pm 0.8$ & $0.52 \pm 0.14$ & $200 \pm 13$ & 50750.54 & & 0.36 & 0.031 \\
\hline \multirow[t]{2}{*}{ DK+ours } & $453.6^{(3)}$ & $3.2 \pm 0.9$ & $-34.7 \pm 0.7$ & $0^{(3)}$ & & 45041.11 & \multirow[t]{2}{*}{-0.26} & 0.13 & 0.001 \\
\hline & $453.6^{(3)}$ & $8.3 \pm 2.8$ & $-34.6 \pm 0.7$ & $0.82 \pm 0.07$ & $344 \pm 16$ & 46799.95 & & 0.20 & 0.005 \\
\hline \multicolumn{10}{|l|}{ Emission wings } \\
\hline $\mathrm{H} \alpha$ wings & $456.0 \pm 6.1$ & $8.6 \pm 1.2$ & $-35.4 \pm 0.70$ & $0^{(3)}$ & & 50376.11 & & & \\
\hline $\mathrm{H} \alpha$ wings & $453.6^{(3)}$ & $8.5 \pm 1.2$ & $-35.4 \pm 0.70$ & $0^{(3)}$ & & 50385.80 & 0.53 & 0.42 & 0.029 \\
\hline $\mathrm{H} \alpha$ wings $^{(4)}$ & $453.6^{(3)}$ & $10.0 \pm 2.3$ & $-36.6 \pm 1.5$ & $0^{(3)}$ & & & & 0.35 & 0.047 \\
\hline
\end{tabular}

Combined orbital solutions $(P=453.6 \pm 0.4$ days $)$.

\begin{tabular}{|c|c|c|c|c|c|c|c|}
\hline Component & $\begin{array}{c}K \\
{\left[\mathrm{~km} \mathrm{~s}^{-1}\right]}\end{array}$ & $\begin{array}{c}\gamma_{0} \\
{\left[\mathrm{~km} \mathrm{~s}^{-1}\right]}\end{array}$ & $e$ & $\begin{array}{c}\omega \\
{[\operatorname{deg}]}\end{array}$ & $\begin{array}{c}T_{0}^{(1)} \\
{[\mathrm{JD} 24 \ldots]}\end{array}$ & $\begin{array}{l}a \sin i \\
{[\mathrm{AU}]}\end{array}$ & $\begin{array}{c}M \sin ^{3} \mathrm{i} \\
{\left[\mathrm{M}_{\odot}\right]}\end{array}$ \\
\hline $\mathrm{DK}+\mathrm{F}+$ ours & $17.1 \pm 0.6$ & $-38.7 \pm 0.4$ & $0^{(3)}$ & & 45612.05 & 0.71 & 0.35 \\
\hline $\mathrm{H} \alpha$ wings & $10.1 \pm 1.2$ & & & & & 0.42 & 0.59 \\
\hline$\overline{\mathrm{DK}+\mathrm{F}+\text { ours }}$ & $17.2 \pm 0.7$ & $-38.7 \pm 0.4$ & $0.04 \pm 0.03$ & $87 \pm 44$ & 45722.37 & 0.72 & 0.35 \\
\hline $\mathrm{H} \alpha$ wings & $10.2 \pm 1.2$ & & & & & 0.42 & 0.59 \\
\hline $\mathrm{DK}+\mathrm{F}+$ ours $^{(4)}$ & $16.4 \pm 1.5$ & $-38.4 \pm 0.8$ & $0^{(3)}$ & & 0.00 & 0.68 & 0.35 \\
\hline $\mathrm{H} \alpha$ wings $^{(4)}$ & $10.4 \pm 1.8$ & & & & & 0.43 & 0.55 \\
\hline
\end{tabular}

Notes: ${ }^{(1)} T_{0}$ is the time of maximum velocity (circular orbits) or the time of periastron passage (elliptical orbits); ${ }^{(2)} \Delta \phi=\left(T_{0}-T_{0, \text { giant }}\right) / P$; (3) assumed; ${ }^{(4)}$ binned data; ${ }^{(5)}$ solutions using only the measurements of the Ti II absorption lines.

of the red giant and assuming that the giant is synchronized. Adopting $v \sin i=11.7 \pm 1.5 \mathrm{~km} \mathrm{~s}^{-1}$ (Zamanov et al. 2007) and $K_{\mathrm{g}}=17.1 \pm 0.6 \mathrm{~km} \mathrm{~s}^{-1}$ (Table 2) results in $q_{\min }=0.68 \pm 0.1$. This lower limit is surprisingly close to the mass ratio derived from the radial velocity curves, and indicates that either the giant is filling its Roche lobe (RL) - the actual $q$ should then be equal to $q_{\min }$ - or that its measured rotational velocity is faster than the synchronized value. The first possibility is favored by theoretically predicted synchronization and circularization timescales for convective stars (Zahn 1977). It is also easier to ensure the high mass tranfer and accretion rate required by the activity and short nova outburst recurrence time of RS Oph. On the other hand, the presence of an RL-filling giant implies that the distance to RS Oph is a factor of 2 larger than generally accepted based on observational evidence (Barry et al. 2008).

The $\mathrm{cF}$ absorption lines do not trace clearly the orbit of the hot component. Any orbital solution leads to a significant eccentricity (see Table 2 ) and the radial velocity curve is shifted by $\sim-0.26 P(0.74 P)$ relative to the $\mathrm{M}$ giant solution instead of $0.5 P$ (see Fig. 3). We think, in agreement with DK94, that in RS Oph the $\mathrm{cF}$ absorption lines are not associated with either binary component, and are most likely formed in the material streaming towards the hot component. We note here that similar complications with the blue absorption system occurred for a few other active symbiotic systems. Mikołajewska \& Kenyon (1996) failed to derive any radial velocity curve and orbital solution for the blue absorption system in Z And. In CI Cyg the radial velocities of the F-type absorption system suggest that their formation region is the material streaming from the giant near the hot component (Mikołajewska \& Mikołajewski 1988), whereas a possible correlation between AR Pav's activity and the departures of the $\mathrm{cF}$ absorption velocities from the circular orbit suggests that this absorption system also may be affected by material streaming towards the hot component, presumably in a region where the stream encounters an accretion disk or an extended envelope around the hot component (Quiroga et al. 2002). We can see in Fig. 3 the departures of the cF-abs radial velocities from the circular orbit of the hot component due to a significant contribution from the stream.

In Figs. 1 and 3 and hereafter we adopt the period of 453.6 days and the origin of the phases corresponds to the inferior conjunction of the M-giant:

$T_{\text {conj }}=$ JD $2445043.54 \pm 5+453.6 \pm 0.4 E$. 


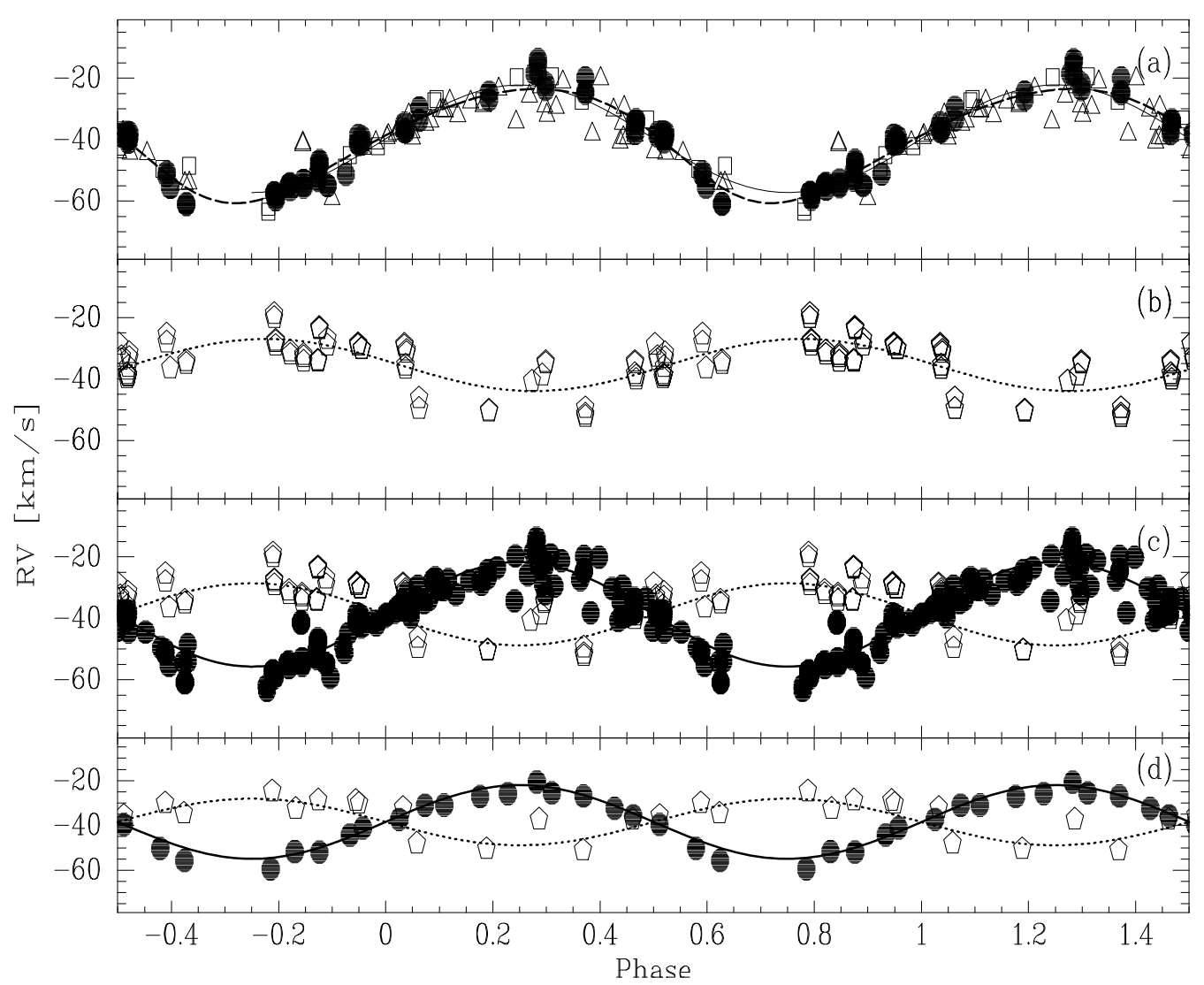

Fig. 1. Radial velocity curves and orbital solutions for RS Oph. The data are phased with a period of 453.6 days and $T_{\text {conj }}=2445043.54 \pm 5$ (the time of the inferior conjunction of the M giant). a) M-giant. Filled circles represent our data and open triangles and squares, the DK94 and FJHS00 data, respectively. The solid line gives the best circular fit and the dashed line gives the eccentric orbit $(e=0.14)$. b) The H $\alpha$ wings (hereafter open symbols) and the best circular solution (dotted line). c) Combined circular solution for the M-giant (filled circles) and the H $\alpha$ wings. d) The same with binned data.
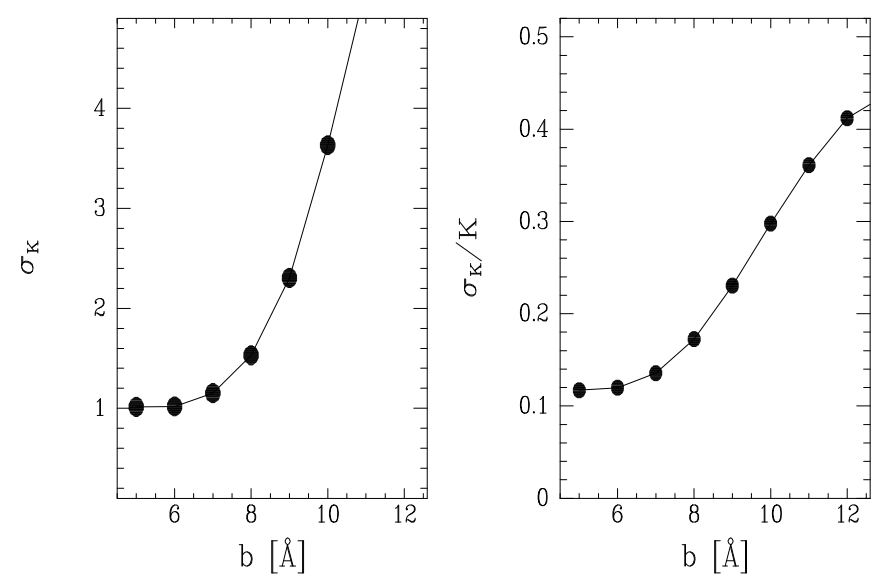

Fig. 2. The standard error of the orbital semi-amplitude $\sigma_{K}$ and the $\sigma_{K} / K$ ratio as a function of the parameter $b$. The best estimation of $K$ is determined by the value of $b$ where $\sigma_{K}$ begins to sharply increase.

\section{RS Oph at quiescence}

Fluctuations in the fluxes of the emission lines and the continuum are observed during the period of quiescence covered by our observations. Several authors have previously reported such behaviour of RS Oph, outside the eruptive episodes (see Anupama \& Mikołajewska 1999, and refences therein).

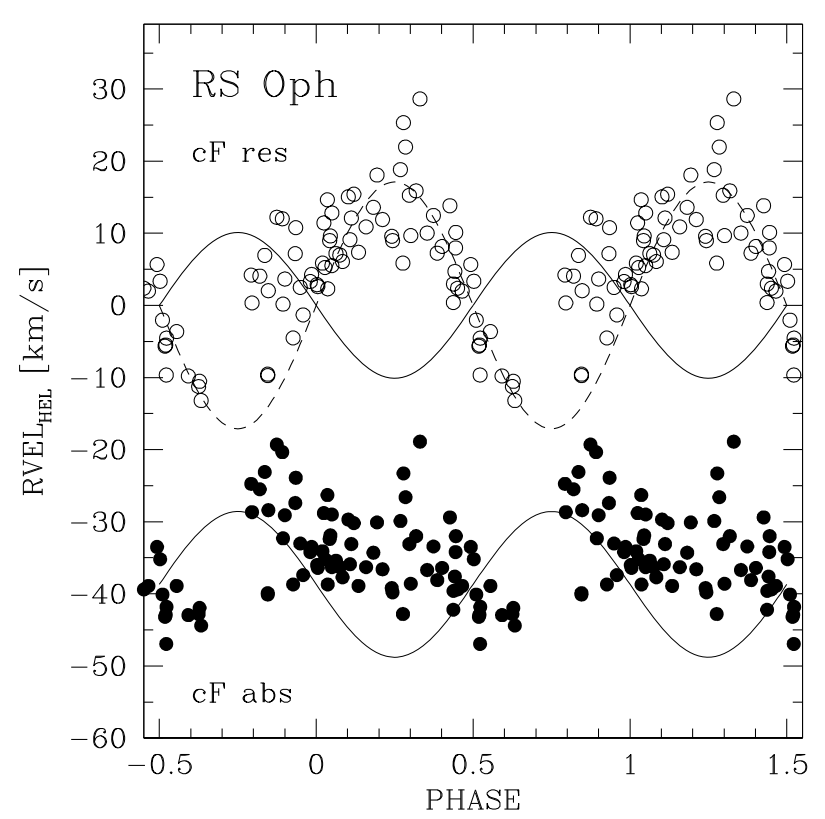

Fig. 3. Phased radial velocities of the cF-absorption lines (dots) and the best circular solution (solid line) for the hot component based on the $\mathrm{H} \alpha$ wings (bottom). The departures of the $\mathrm{cF}$-abs radial velocities from the circular orbit of the hot component are due to a significant contribution from the gaseous stream between both stars. The residuals $(\mathrm{O}-\mathrm{C})$ are represented by empty circles whereas the solid and dashed lines mark the best circular solution for the hot and cool component, respectively (top). 

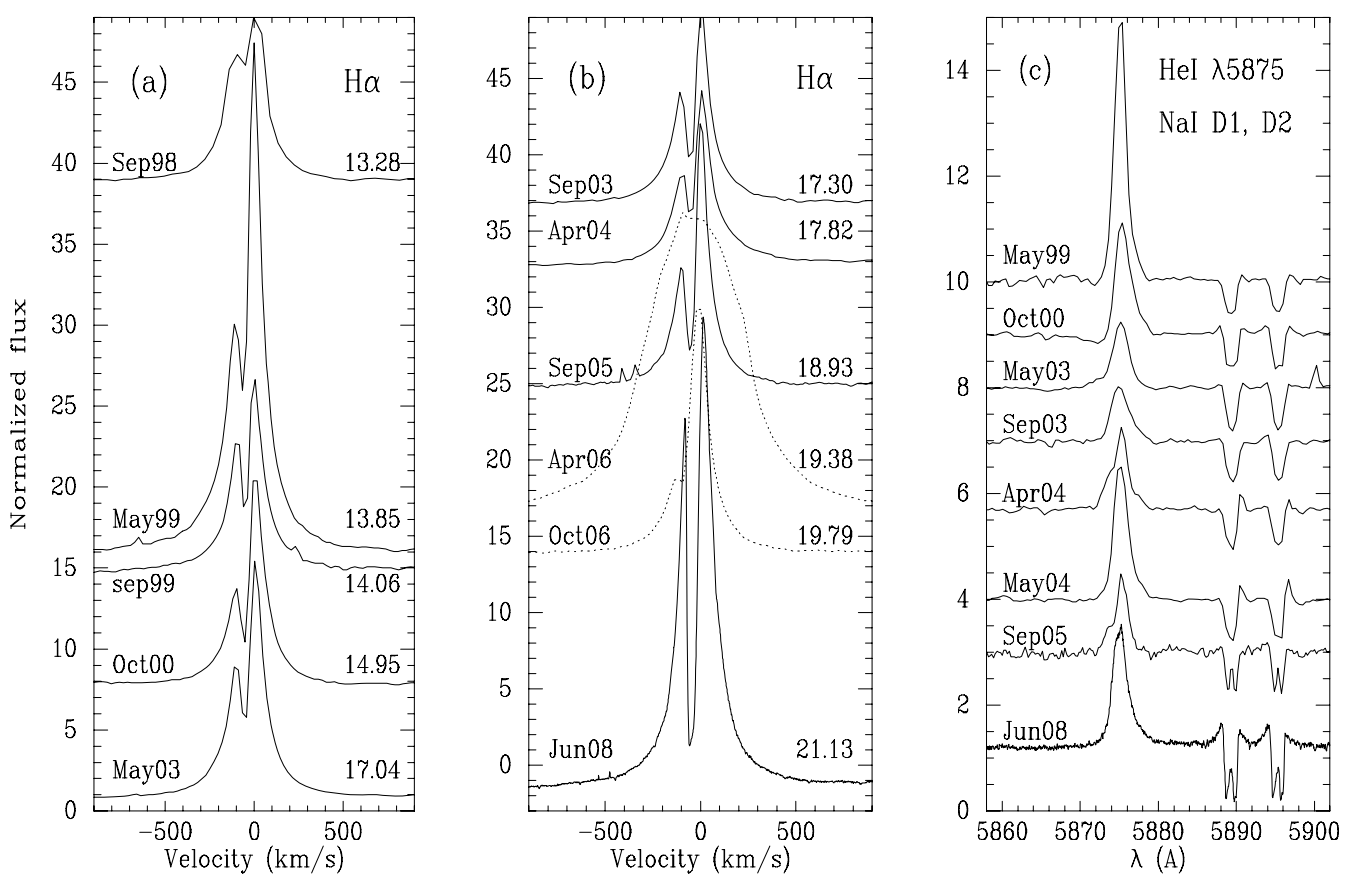

Fig. 4. Panels a) and b): profiles of $\mathrm{H} \alpha$. At quiescence the broad emission is cut by a central absorption which disappears during the outburst (dotted profile). Panel c): profile variations of He I $\lambda 5875$ and Na I doublet during the quiescent period. Blueshifted broad components in He I are present on April 2004 and September 2005. The Na I doublet shows weak emission components and double structure in absorption.

Table 3. Mean values of radial velocities and equivalent widths of the central absorptions in $\mathrm{H} \alpha$ and $\mathrm{H} \beta$.

\begin{tabular}{lccccc}
\hline \hline $\begin{array}{l}\text { JD } \\
2450000+\end{array}$ & $\begin{array}{c}\text { Phases } \\
\text { (days) }\end{array}$ & $\begin{array}{r}\mathrm{H} \alpha \\
\mathrm{RV}\left(\mathrm{km} \mathrm{s}^{-1}\right)\end{array}$ & eqw & $\begin{array}{r}\mathrm{RV}\left(\mathrm{km} \mathrm{s}^{-1}\right) \\
\text { eqw }\end{array}$ \\
\hline 1069.6 & 13.285 & -57.7 & 0.46 & -25.6 & 0.43 \\
1324.7 & 13.847 & -56.6 & 0.59 & -47.9 & 0.57 \\
1422.6 & 14.063 & -50.2 & 0.53 & -43.0 & 0.72 \\
1629.9 & 14.518 & -48.8 & 0.71 & -45.0 & 0.60 \\
1754.7 & 14.795 & -52.3 & 0.70 & -46.9 & 0.58 \\
1790.6 & 14.874 & -52.4 & 0.73 & -45.1 & 0.61 \\
1791.6 & 14.877 & -50.0 & 0.67 & & \\
1824.5 & 14.949 & -50.7 & 0.74 & -47.1 & 0.66 \\
1827.5 & 14.956 & -52.7 & 0.72 & & \\
2016.9 & 15.373 & -48.4 & 0.75 & -45.2 & 0.65 \\
2059.9 & 15.468 & -43.8 & 0.59 & -47.8 & 0.60 \\
2132.6 & 15.628 & -48.1 & 0.62 & -54.7 & 0.64 \\
2388.9 & 16.193 & -42.9 & 0.86 & -43.4 & \\
2531.6 & 16.508 & -38.8 & 0.77 & & \\
2770.9 & 17.036 & -48.4 & 0.63 & -48.5 & 0.76 \\
2771.8 & 17.038 & -44.4 & 0.76 & -41.1 & 0.86 \\
2890.5 & 17.299 & -50.1 & 0.86 & & \\
3126.8 & 17.820 & -48.4 & 0.68 & -40.8 & 0.56 \\
3127.8 & 17.822 & -47.3 & 0.83 & & \\
3476.9 & 18.592 & -43.8 & 0.93 & -42.9 & 0.75 \\
3628.6 & 18.926 & -51.1 & 0.92 & -48.2 & 0.79 \\
4600.8 & 21.070 & -60.0 & 0.94 & -54.5 & 0.88 \\
4627.8 & 21.129 & -43.6 & 1.07 & -35.2 & 0.95 \\
\hline
\end{tabular}

The spectra present essentially emission lines of H I, He I and [OI] at $6300 \AA$. In the observed members of the Balmer series, the broad emission is cut by a central absorption with a red peak always stronger than the blue one. Figure 4, left and central panels, show $\mathrm{H} \alpha$ profiles for several epochs. At quiescence the central absorption remains present although with variable intensity over the whole orbital cycle, whereas it disappears during the eruption, and it is weakly visible again in October 2006. The radial velocity of the central absorption of $\mathrm{H} \alpha$ varies between -60 and $-40 \mathrm{~km} \mathrm{~s}^{-1}$. As it is shown in Fig. 5a this variation does not follow the motion of any stellar component of the system but Figs. $5 \mathrm{~b}$ and $\mathrm{c}$ show changes of both the radial velocities and the equivalent widths with the different cycles of the binary, in the sense that the mean velocity was increasing until cycle 16 (May 2003) and then decreasing, whereas the equivalent widths were monotonically increasing.

The profile of $\mathrm{H} \beta$ also presents a double peak structure but the radial velocity of the central absorption is rather constant, without a clear variation with orbital phase or orbital cycle (see Figs. 5d-f).

Significant changes in the radial velocity of the central absorption in both $\mathrm{H} \alpha$ and $\mathrm{H} \beta$ were observed in the higher resolution Feros spectra between May and June 2008 (asterisks in Fig. 5). An explanation of this behaviour correlating the radial velocities with the flickering activity and the accretion processes in RS Oph is in progress.

Broad emission components were detected in the emission line He I $\lambda 5875$ during April 2004 and September 2005 (see Fig. 4, right panel) at velocities of the order of $\sim 100 \mathrm{~km} \mathrm{~s}^{-1}$. The intensity of the He I emission lines and the presence of these broad components change quickly with a time scale of one or two days.

Figure 6 presents the $\mathrm{H} \alpha$ fluxes against the $m_{4800}$ magnitudes calculated from our spectra. The figure shows that a clear correlation between both data exists, confirming that the emission line variability is correlated with the hot component activity (Anupama \& Mikołajewska 1999).

\subsection{Presence of the Lil $\lambda 6707$ absorption line}

One unsolved problem in astrophysics is the origin of lithium and other light elements in the universe. How and when lithium is depleted and an understanding of the processes controlling 

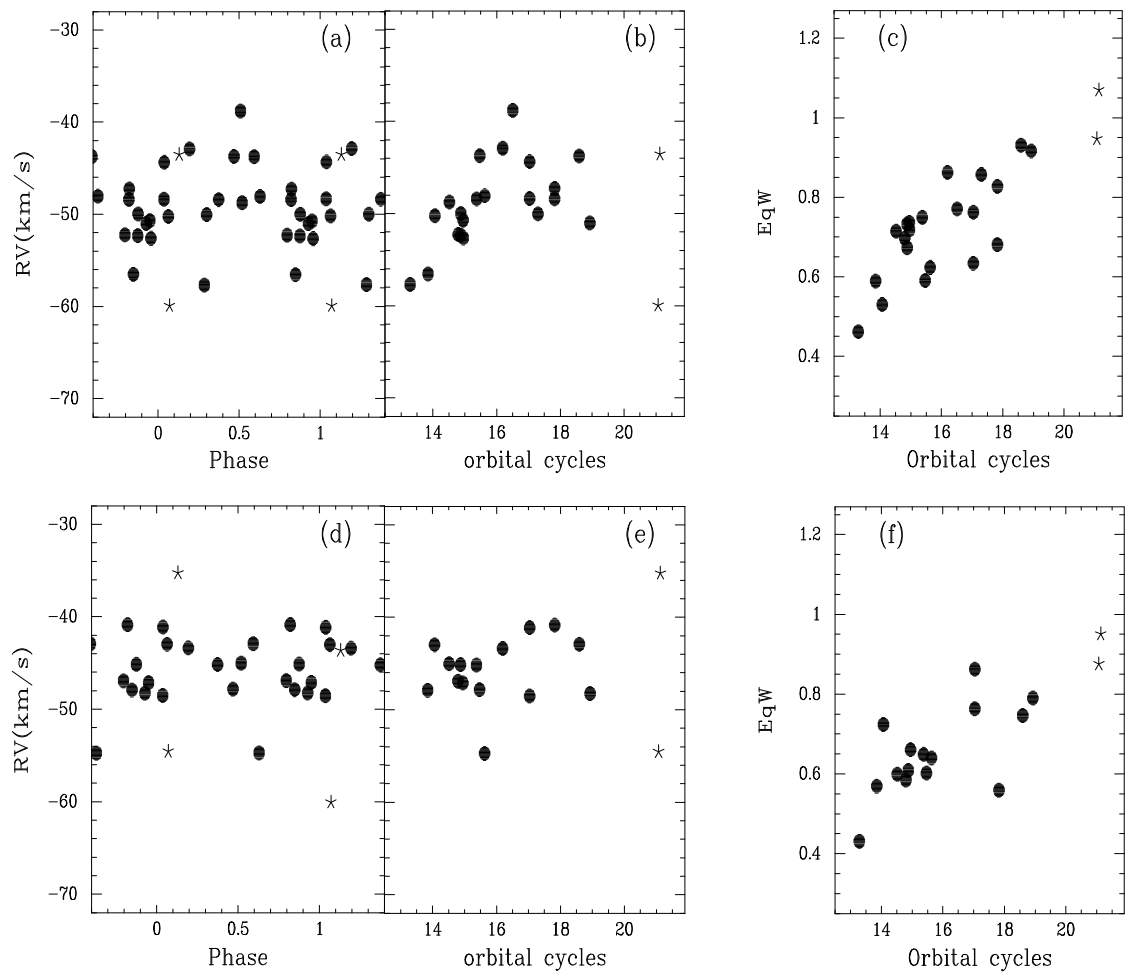

Fig. 5. Radial velocities and equivalent widths of the central absorptions of $\mathrm{H} \alpha$ (panels a)-c)) and $\mathrm{H} \beta$ (panels d)-f)) as a function of the orbital phases and the orbital cycles, respectively. Asterisks correspond to the Feros spectra.

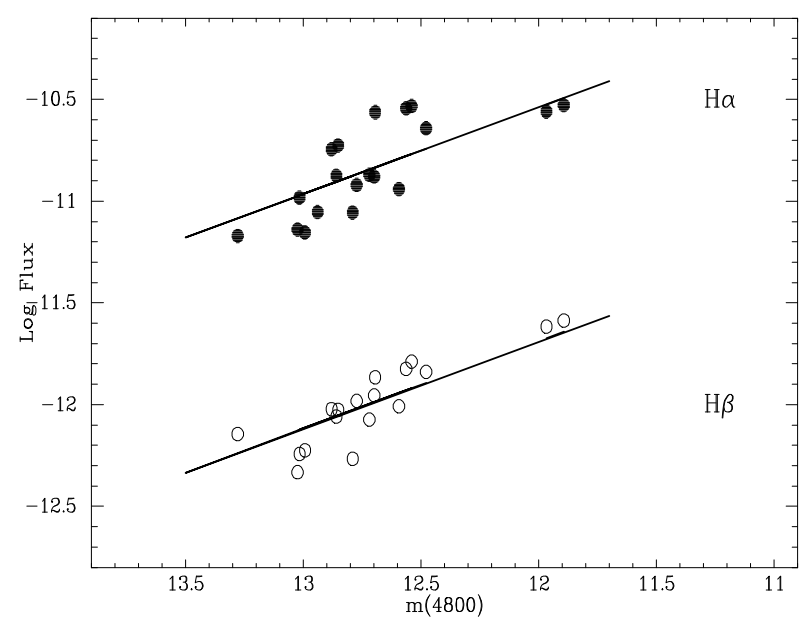

Fig. 6. Correlation of the $\mathrm{H} \alpha$ and $\mathrm{H} \beta$ emission line fluxes with the magnitudes at $4800 \AA$ measured from our spectra.

lithium production are still not completely resolved. The synthesis of ${ }^{7} \mathrm{Li}$ in explosive hydrogen burning, in particular in accreting $(\mathrm{CO}$ or $\mathrm{ONe})$ white dwarfs exploding as classical novae has been studied by several authors (see Jordi 2005, and references therein) and novae are predicted to be important sources of Galactic lithium.

However the observation of ${ }^{7} \mathrm{Li}$ in the ejecta of a nova outburst has been rather elusive. The Li I $\lambda 6708$ line has been detected in the recurrent symbiotic novae $\mathrm{T}$ CrB (Shabhaz et al. 1999) and RS Oph (Wallerstein et al. 2006). Wallerstein et al.(2008) have recently derived $\mathrm{Li}$ abundances in these stars, $\log N(\mathrm{Li})=1.2$ for RS Oph and 0.8 for $\mathrm{T} \mathrm{CrB}$, which are close to being solar. These Li abundances are however significantly higher than those determined for most single $\mathrm{K}$ and $\mathrm{M}$ giants. Such high Li abundances are common in late-type secondaries in neutron and black hole binaries (e.g. Martin et al. 1994) but extremely rare in the symbiotic giants. In fact, the Li enhancement is thus far detected only in the symbiotic Mira V407 Cyg, where it can probably be explained as a consequence of hot bottom burning, which occurs in stars with initial masses in the range 4$6 M_{\odot}$ (Tatarnikova et al. 2003). Such an explanation is, however, unlikely in the case of low mass, $\lesssim 1 M_{\odot}$, nonpulsating giants in RS Oph and T CrB.

Our spectra of RS Oph show the presence of the Li I line, and the radial velocities of this line fit very well to the M-giant radial velocity curve (see right panel Fig. 7). The identification of the weak and narrow absorption line at 6706-6708 $\AA$ with $\mathrm{Li}$ I $\lambda 6708$ is unambiguous since no other neutral element following the giant motion has a strong transition at that wavelength. In addition, the presence of this line is clearly seen in the higher resolution Feros spectra. The feature was observed in all our spectra, except in April 2006 where all the absorption lines were overwhelmed by very strong blue continuum from the nova outburst.

This result indicates that, regardless of the mechanism involved to generate lithium, it should operate within the cool giant atmosphere. The observed Li would be the initial Li (which is observed in non convective stars) if some process operating in such binary systems as RS Oph and T CrB, e.g. delayed onset of convection or a lack of differential rotation due to tidal locking, can inhibit lithium depletion (Shabhaz et al. 1999).

Wallerstein et al. (2008) offered another possibility for the presence of $\mathrm{Li}$. They suggested that it is freshly created in the interior and convected to the surface. They also noted that some $\mathrm{G}$ and K-type giants have very high atmospheric Li abundances. In the case of these Li-rich giants, Charbonnel \& Balachandran (2000) identified two distinct episodes of Li production occuring 

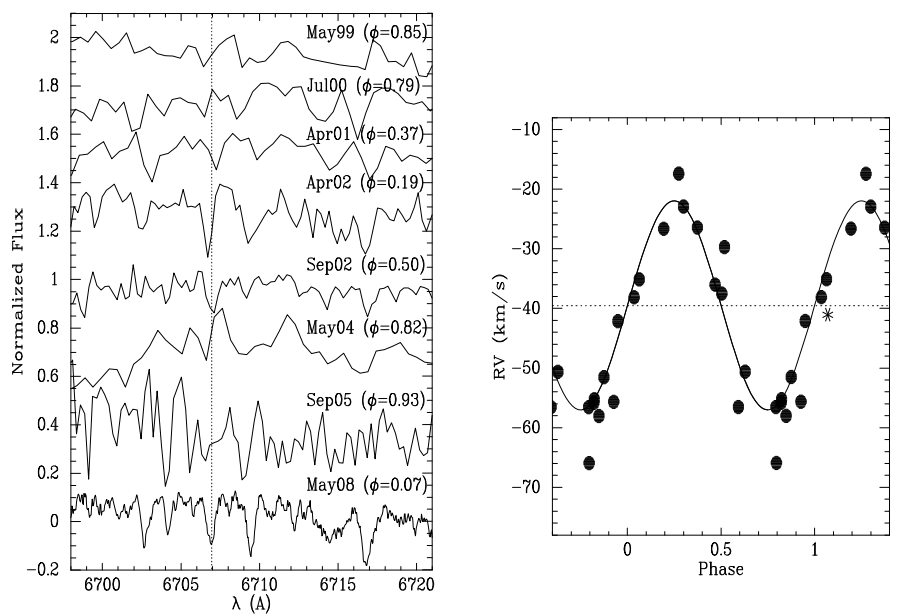

Fig. 7. (Left) Presence of the Li I $\lambda 6707$ absorption line in RS Oph during quiescence. The dotted line indicates the position of the line corrected by the baricentric velocity of the binary. (Right) The radial velocities of Li I following the M-giant motion. Filled circles, Casleo data and asterisk, Feros data. The solid line gives the circular solution for the M-giant, as in Fig. 1.

in advanced evolutionary phases depending upon the mass of the star. Low-mass RGB stars which later undergo a helium flash produce $\mathrm{Li}$ at the start of the red giant luminosity bump phase whereas in intermediate-mass stars the Li-rich phase occurs when the convective envelope deepens at the base of the AGB. The position of the cool giant of $\mathrm{T} \mathrm{CrB}$ in the HR diagram is consistent with a low-mass, $\sim 1 M_{\odot}$, star at the top of the RGB whereas the location of RS Oph is consistent with such a giant only if its metallicity is significantly subsolar (Mikołajewska 2008), and in both cases the stars are located far from the RGB bump. On the other hand, Wallerstein et al. (2008) found a normal, nearly solar, ratio of metals to hydrogen in both RS Oph and $\mathrm{T} \mathrm{CrB}$, and they concluded that the giant component has not yet lost sufficient mass to make its atmosphere deficient in hydrogen. It seems therefore unlikely that the initial mass of the $\mathrm{M}$ giant was significantly greater than its current estimate of $0.68-0.8 M_{\odot}$.

An alternative explanation is the pollution of the giant by the nova ejecta. This explanation has, however, some weak points. In particular, the red giant should very efficiently accrete the Li-rich material ejected at very high velocity (a few $1000 \mathrm{~km} \mathrm{~s}^{-1}$ !) to be significantly polluted. Moreover there is also serious controversy about the production of Li during such an explosion.

\section{RS Oph in 2006}

High-dispersion spectra of RS Oph were taken at CASLEO with the same instrumental configuration on 2006 April 7-8 and October 12, that is, 54-55 and 240 days following the explosion. The spectra of April show broad emission lines of hydrogen recombination lines, together with He I, He II, Fe II, N III, [O I] $\lambda 6300,[\mathrm{O}$ III] $\lambda 5007,[\mathrm{~N} \mathrm{II}] \lambda 5754$ and the Raman band at $\lambda 6825 \AA$. The $\mathrm{cF}$ absorption system is not observed in April 2006. Very narrow emission components are seen on top of the broad emission components.

Strong coronal emission lines such as [Fe XIV] $\lambda 5305$, [A X] $\lambda 5535$ and [Fe X] $\lambda 6375$ (Fig. 8) are also present, showing the same structure in the profiles. Our measures of the integrated fluxes, the radial velocity of the emission line components and
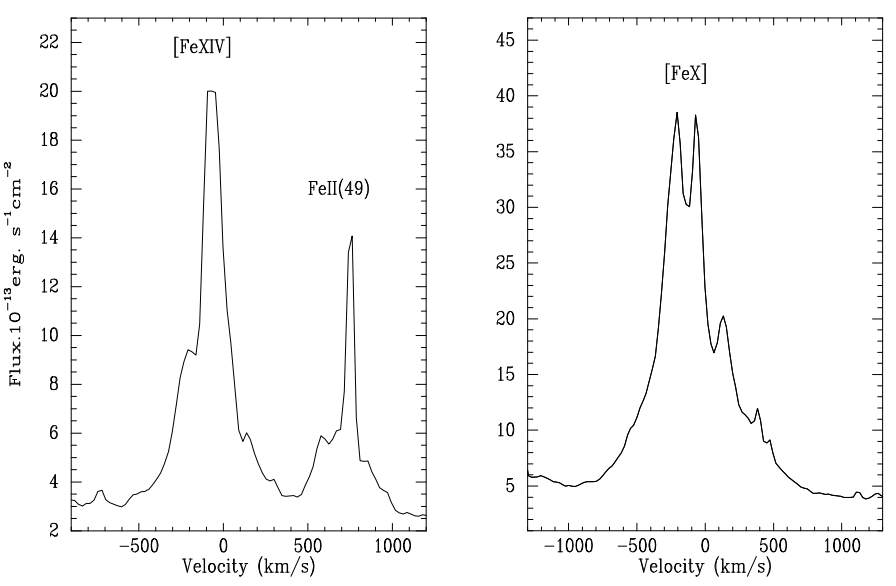

Fig. 8. Observed coronal lines in RS Oph on April 2006.

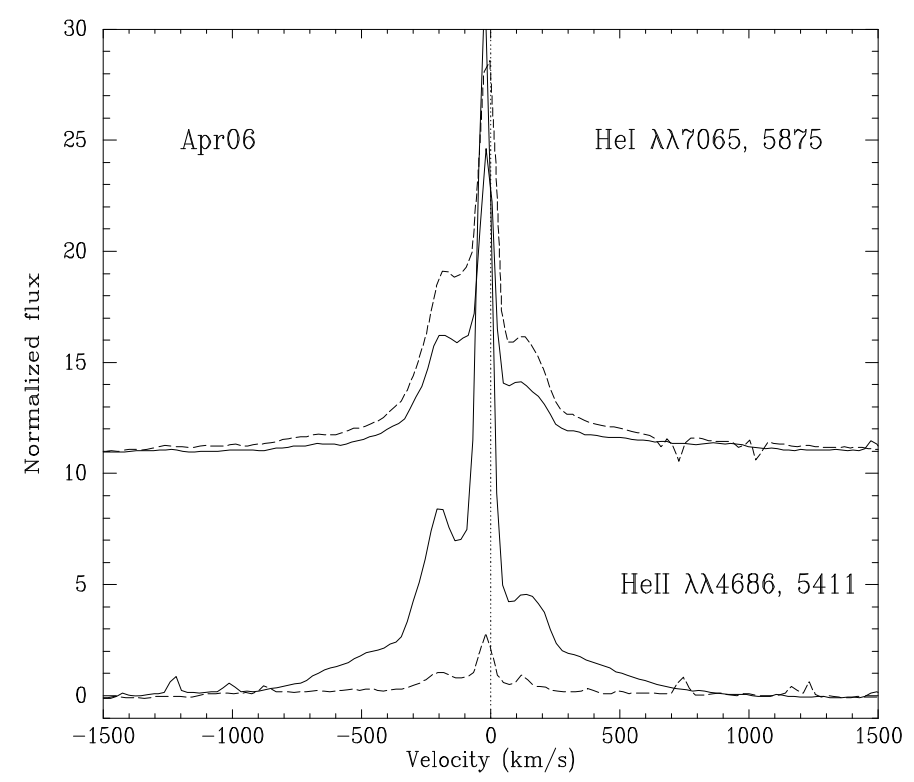

Fig. 9. The He I and He II profiles showing a broad pedestal with the strong narrow component at -15 and $-23 \mathrm{~km} \mathrm{~s}^{-1}$ respectively. Two separate emission components of higher radial velocity at -200 and $+150 \mathrm{~km} \mathrm{~s}^{-1}$ are observed, the blue component being stronger than the red one.

the full width at zero intensity (FWZI) of several stronger lines are shown in Table 4.

Most of the emission lines present a strong and narrow component with a radial velocity between -14 and $-25 \mathrm{~km} \mathrm{~s}^{-1}$ and two other components extended to the blue and the red side. In the cases of the $[\mathrm{NII}]$ and $[\mathrm{O} \mathrm{III}]$ emission lines and the coronal lines, the radial velocity of the narrow component is more negative ( -40 and $-50 \mathrm{~km} \mathrm{~s}^{-1}$ ).

We can see in Fig. 9 the He I $\lambda \lambda 5875,7065$ and He II $\lambda 4686$, 5411 profiles showing a broad pedestal with the strong narrow component at -15 and $-23 \mathrm{~km} \mathrm{~s}^{-1}$ respectively. Two separate emission components of higher radial velocity are observed at $\sim-200$ and $+150 \mathrm{~km} \mathrm{~s}^{-1}$ with the blue component stronger than the red one. An explanation based on a bipolar gas outflow may be applicable to the presence of these components with expanding and receding radial velocities.

On October 2006, the RS Oph spectrum is slowly restoring its quiescent characteristics. The cF-absorption system, absent in April 2006, has reappeared. The intensity of the emission lines decreases, the coronal lines are not observed and the blue and 
Table 4. Emission lines of RS Oph on April 2006.

\begin{tabular}{|c|c|c|c|c|c|c|}
\hline \multirow[t]{2}{*}{ Species } & \multirow{2}{*}{$\begin{array}{c}\lambda \\
{[\AA]}\end{array}$} & \multirow{2}{*}{$\begin{array}{l}\text { Flux }\left(10^{-12}\right) \\
\left(\operatorname{erg~s}^{-1} \mathrm{~cm}^{-2}\right)\end{array}$} & \multicolumn{3}{|c|}{$\mathrm{RV}\left(\mathrm{km} \mathrm{s}^{-1}\right)$} & \multirow{2}{*}{$\begin{array}{c}\text { FWZI } \\
\left(\mathrm{km} \mathrm{s}^{-1}\right)\end{array}$} \\
\hline & & & narrow & blue & red & \\
\hline \multicolumn{7}{|c|}{ (ciso } \\
\hline $\mathrm{H} \beta$ & 4861 & 59.1 & -21 & -38 & & 2500 \\
\hline $\mathrm{H} \alpha$ & 6562 & 460 & -47 & & & 3840 \\
\hline $\mathrm{P} 23$ & 8345 & 0.6 & -19 & & & \\
\hline P22 & 8359 & 1.0 & -20 & & & \\
\hline P21 & 8374 & 1.0 & -23 & & & \\
\hline P20 & 8392 & 1.3 & -20 & & & \\
\hline P19 & 8413 & 2.2 & -16 & & & \\
\hline \multicolumn{7}{|l|}{$\mathrm{He} \mathrm{I}$} \\
\hline & 4471 & 2.6 & -19 & -205 & 81 & 1100 \\
\hline & 5875 & 25.4 & -14 & -194 & 155 & 1950 \\
\hline & 6678 & 9.4 & -15 & -208 & & 740 \\
\hline & 7065 & 28.1 & -17 & -196 & 156 & 1400 \\
\hline \multicolumn{7}{|l|}{ He II } \\
\hline & 4686 & 26.7 & -25 & -209 & 164 & 1680 \\
\hline & 5411 & 3.4 & -21 & -208 & 122 & 1050 \\
\hline \multicolumn{7}{|l|}{ Fe II } \\
\hline M27 & 4233 & 1.9 & -20 & -186 & & 800 \\
\hline M38 & 4583 & 1.7 & -20 & $-272:$ & $77 \mathrm{w}$ & 650 \\
\hline M42 & 5169 & 2.3 & -20 & -121 & $123 w$ & 580 \\
\hline M49 & 5234 & 1.4 & -22 & -124 & 134 & 720 \\
\hline M49 & 5316 & 2.4 & -18 & -195 & $88 w$ & 580 \\
\hline M74 & 6247 & 0.8 & -22 & -141 & 47 & 514 \\
\hline M73 & 7711 & 1.7 & -24 & -122 & 181 & 717 \\
\hline Raman band & 6825 & 5.1 & & & & 1470 \\
\hline \multicolumn{7}{|l|}{ Forbidden lines } \\
\hline$[\mathrm{OI}]$ & 6300 & 0.6 & -15 & -94 & 90 & 350 \\
\hline [N II] & 5754 & 4.6 & -42 & & 81 & 925 \\
\hline [O III] & 5007 & 1.7 & -40 & -104 & & 320 \\
\hline \multicolumn{7}{|l|}{ Coronal lines } \\
\hline [Fe XIV] & 5303 & 7.0 & -52 & -220 & $163 \mathrm{w}$ & 900 \\
\hline$[\mathrm{A} \mathrm{X}]$ & $5535(\star)$ & 7.5 & -13 & -282 & $100 \mathrm{w}$ & 1930 \\
\hline$[\mathrm{Fe} \mathrm{X}]$ & 6375 & 35.1 & -40 & -188 & 166 & 1500 \\
\hline
\end{tabular}

w: very weak line.

( $\star$ ): blend with Fe II $\lambda 5534.9$.

red components of the He I lines have disappeared. $\mathrm{H} \alpha$ has recovered its pre-outburst profile, in particular the emission wings are narrower than in April 2006 and the emission is cut by an incipient central absorption (see Fig. 4). A very weak He II $\lambda 4686$ line is still observed, although its flux has decreased by a factor of $\sim 90$ with respect to that of April 2006. This profile preserves the strong emission component at $-46 \mathrm{~km} \mathrm{~s}^{-1}$ and two expanding components at -214 and $+192 \mathrm{~km} \mathrm{~s}^{-1}$, with the red component being stronger than the blue one, that is, in the opposite sense of that of April (Fig. 10, left panel).

\section{Conclusions}

1. We have re-determined the spectroscopic orbits of RS Oph based on the radial velocity curve of the M-type absorption lines at wavelengths longer than $6000 \AA$. As the HI broad emission wings seem to follow the hot component motion, combined orbital solutions for both components were also determined with a period of 453.6 days, very similar to those given by FJHS00. We conclude, as DK94 do, that the cF-type absorption lines are not associated with either binary component, and are most likely formed in the material streaming towards the hot component.

Assuming a massive white dwarf as the hot component of the system $\left(M_{\mathrm{h}}=1.2-1.4 M_{\odot}\right)$ the red giant mass results in $M_{\mathrm{g}}=0.68-0.80 M_{\odot}$ and the orbit inclination, $i=49^{\circ}-52^{\circ}$.
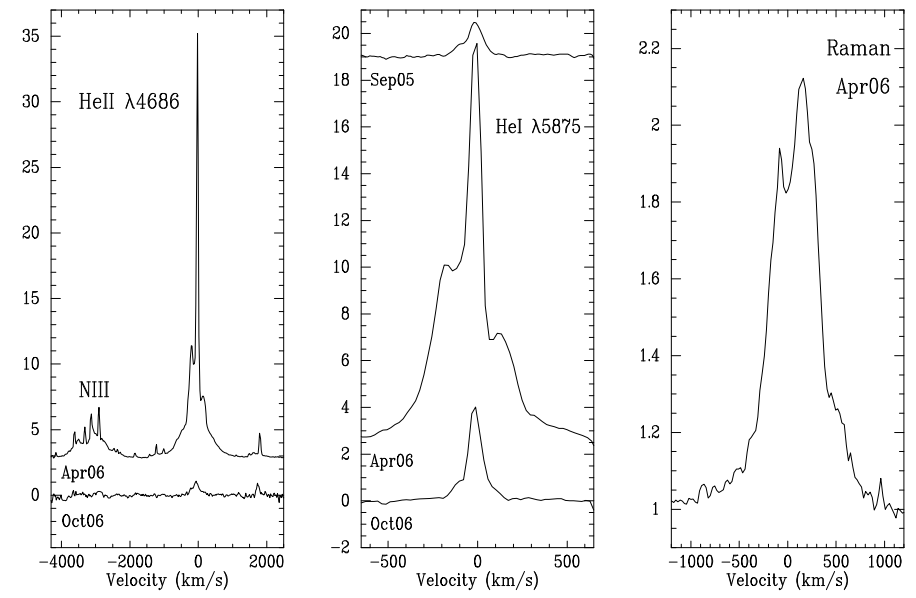

Fig. 10. Left panel: NIII emission lines observed in April 2006. The He II $\lambda 4686$ is still detected on 2006 October 12. Middle panel: intensity and profile variations of $\mathrm{He} \mathrm{I} \lambda 5875$ before and after explosion. Right panel: the scattering Raman band at $6825 \mathrm{~A}$, which does not appear at all during the quiescent phase, is present as a strong emission profile in April 2006. This feature is not observed in October 2006.

2. During the quiescent period of our observations the spectra of RS Oph show variability in the fluxes of the emission lines and in the continuum. A correlation of the H I and He I 
emission line fluxes with the monochromatic magnitudes at $4800 \AA$ was obtained, indicating that the hot component activity is responsible for those flux variations.

3. Our spectra of RS Oph reveal the presence of the Li I 6708 line, and the radial velocities of this line fit the M-giant radial velocity curve very well. This result indicates that, whatever the mechanism involved to generate lithium would be, it should operate within the cool giant atmosphere. An alternative explanation is the pollution of the giant by the nova ejecta but the red giant in this case should very efficiently accrete the Li-rich material ejected at very high velocity to be significantly polluted.

4. We present the characteristics of the spectra around 5455 and 240 days after the outburst of February 2006. On April 2006 most of the emission lines present a broad pedestal with a strong and narrow component at about $-20 \mathrm{~km} \mathrm{~s}^{-1}$ and two other extended emission components at -200 and $+150 \mathrm{~km} \mathrm{~s}^{-1}$. These components could originate in a bipolar gas outflow supporting the model of a bipolar shock-heated shell expanding through the cool component wind and perpendicular to the orbital plane of the binary.

5. Our observations indicate that the $\mathrm{cF}$ system was disrupted during the outburst. The $\mathrm{cF}$ absorption lines were not observed in our spectra in April 2006 and Zamanov et al. (2006) reported the absence of flickering in June 2006, then the accretion was not yet resumed immediately after the outburst. Both the $\mathrm{cF}$ system and the flickering (Worters et al. 2007) were observed again about 240 days after the outburst, which is consistent with the resumption of accretion in RS Oph.

Acknowledgements. We are deeply indebted to Drs. N. Morrell and R. Barbá for obtaining the Feros spectrograms at ESO, La Silla (Chile). We also thank the anonymous referee for very valuable comments. This research was partly supported by Polish research grants Nos. 1P03D01727, and N203 395534. The CCD and data acquisition system at CASLEO has been partly finance by R. M. Rich through US NSF grant AST-90-15827.

\section{References}

Anupama, G. C., \& Mikołajewska, J. 1999, A\&A ,344, 177

Barry, R. K., Mukai, K., Sokoloski, J., et al. 2008, in the Proceedings of the Meeting, RS Ophiuchi (2006), ed. N. Evans, M. Bode, \& Tim O’Brien, ASP Conf. Ser., 401

Brandi, E., Mikołajewska, J., Quiroga, C., et al. 2005, A\&A, 440, 239

Charbonnel, C., \& Balachandran, S. C. 2000, A\&A, 359, 563

Dobrzycka, D., \& Kenyon, S. 1994, AJ, 108, 2259

Fekel, F. C., Joyce, R. R., Hinkle, K., \& Skrutskie, M. F. 2000, AJ, 119, 1375

Garcia, M. 1986, AJ, 91, 1400

Hamuy, M., Walker, A. R., Suntzeff, N. B., et al. 1992, PASP, 104, 533

Hamuy, M., Suntzeff, N. B., Heathcote, S. R., et al. 1994, PASP, 106, 566

Jordi, J. 2005, NuPhA, Nucl. Phys. A, 758, 713

Lucy, L. B., \& Sweeney, M. A. 1971, AJ, 76, 544

Martin, E. L., Rebolo, R., Casares, J., \& Charles, P. A. 1994, ApJ, 435, 791

Mikołajewska, J. 2008, in the Proceedings of the Meeting: RS Ophiuchi (2006),

ed. N. Evans, M. Bode, \& Tim O'Brien, ASP Conf. Ser., 401

Mikołajewska, J., \& Kenyon, S. J. 1992, AJ, 103, 579

Mikołajewska, J., \& Kenyon, S. J. 1996, AJ, 112, 1659

Mikołajewska, J., \& Mikołajewski, M. 1988, in The Symbiotic Phenomenon, ed. J. Mikołajewska, M. Friedjung, S. J. Kenyon, \& R. Viotti (Dordrecht: Kluwer), 187

Oppenheimer, B., \& Mattei, J. A. 1993, BAAS, 25, 1378

Quiroga, C., Mikołajewska, J., Brandi, E., et al. 2002, A\&A, 387, 139

Schneider, D. P., \& Young, P. 1980, ApJ, 238, 946

Shabhaz, T., Hauschildt, P., Naylor, T., \& Ringwald, F. 1999, MNRAS, 306, 675

Tatarnikova, A. A., Marese, P. M., Munari, U., et al. 2003, MNRAS 344, 1233

Wallerstein, G., Harrison, T., \& Munari, U. 2006, BAAS, 38, 1160

Wallerstein, G., Harrison, T., Munari, U., \& Vanture, A. 2008, PASP, 120, 492

Worters, H. L., Eyres, S. P. S., Bromage, G. E., \& Osborne, J. P. 2007, MNRAS, 379,1557

Zahn, J.-P. 1977, A\&A, 57, 383

Zamanov, R., Panov, K., Boer, M., \& Coroller, H., Le 2006, The Astronomer's Telegram, 832

Zamanov, R. K., Bode, M. F., Melo, C. H. F., et al. 2007, MNRAS, 380, 1053 